\title{
Hybrid Automata System for Emotion Embodiment in Upper Extremity Rehabilitation System
}

\author{
Shahrul Naim Sidek ${ }^{*}$, Aimi Shazwani Ghazali ${ }^{*}$, Saodah Wok ${ }^{*}$
}

\begin{abstract}
This paper highlights the embodiment of emotions which is structured using hybrid automata framework. Each initialization value, modes, condition for each mode, guard conditions, and transition between modes of the automata model designed for robot-assisted rehabilitation system are elaborated. A structured experimental setup was designed to invoke the emotion of the subject and to test the integration between detected emotion with the rehabilitation platform. The evaluation of the hybrid automata proposed is based on real time application. The result demonstrates the efficacy of hybrid automata approach in the rehabilitation application where emotion of the subject is taken into consideration in deploying suitable upper extremity rehabilitation tasks for Human Machine Interaction (HMI) applications.
\end{abstract}

Keywords: emotion embodiment, hybrid automata, HMI, rehabilitation platform

\section{Introduction}

Hybrid automata is a mixed, formal model of system consisting of discrete and continuous elements [1]. The system embeds the digital/discrete real-time component in ana$\log /$ continuous environments used for modeling and design of embedded system [2]. The hybrid automata model has been broadly utilized in various fields of applications, especially in information technology and control engineering. For example in mobile robot control, hybrid cellular automata controller is used in the modeling of non-convex obstacle detection and avoidance for robot exploration in the specific environment [3]. An integrated motor-transmission power-train for an automatic gear shift in electric vehicle also proves that hybrid automata model is able to give a quality result in shifting process which leads to a better vehicle performance [4]. Other applications are modelling of human driving behavior [5], longitudinal control of intelligent vehicle [6], Cyber-Physical Systems (CPS) control [7], and in a stage stunt system [8]. The hybrid system is operated when a specific event is triggered; where each event or mode is treated as separate, continuous systems [9]. In rehabilitation robot application, the mode of the system operation is based on the subject muscle recovery level that is accessed based on the percentage of effort the subject exerts against normal averaged muscle strength [10].

In the field of emotion embodiment especially found in the HMI systems, there are quite a few formalisms found in the literature survey in constructing the emotion recognition module for the

\footnotetext{
* International Islamic University Malaysia, Kuala Lumpur, Malaysia
} 
systems. For instance, fuzzy min-max neural network was designated as the control scheme for the Kaist Motion Expressive Robot (KaMERo) mend to control the robot responds differently to a variety of emotions so to increase the degree of believability of the robot [11]. In [12], Nao robot was programmed with hybrid controller to react accordingly to the subject emotion based on the EOG signal and event-related potential (ERP) signal generated by the subject. A rehabilitation platform called PHYSIOBOT [13] was designed to give psychophysiological feedbacks in a cooperative manner where a virtual neuro-rehabilitation task that is controlled by Fuzzy logic inference system (FIS) is used in giving several commands from the psychophysiological data input that is translated into 0 for no assistance and 1 for full assistance .

The aim of this paper is to present a way to embody the emotion recognizer module into the controller of a dedicated robot assisted rehabilitation platform by using hybrid automata architecture. Emotion detected from the subject is treated as discrete event input that triggers different set of continuous rehabilitation tasks.

\section{System Description}

Figure 1 illustrates the block diagram of robot-assisted rehabilitation system with emotion recognition module. It consists of three major blocks which are emotion recognition system, hybrid automata governing the scheme of controller switching and rehabilitation platform. In the next sections, each of the blocks is discussed in detailed.

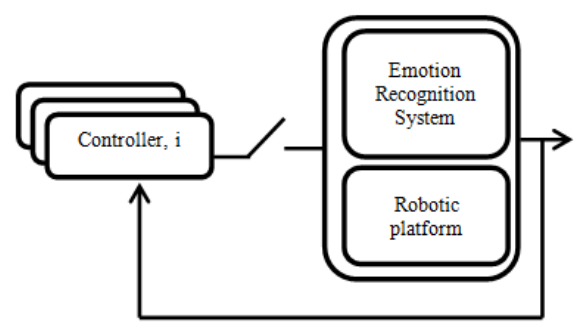

Figure 1: Block Diagram of Rehabilitation Robot with Emotion Embodiment System

\subsection{Emotion Recognition System}

The emotion model developed for the system is based on Bayesian network model. A structured experiment was conducted to invoke the intended emotions. In particular, a series of ten minutes audio visual stimuli are given to the subject before the radiated pattern of electromagnetic (EM) fields from the subject is captured and recorded [14]. A handheld device called Resonant Field Imaging $\left(\mathrm{RFI}^{\mathrm{TM}}\right)$ is used in capturing the EM wave signal. The signals are measured from 10 points on the body that are left and right palms, thighs, forearms, arms, and sides of head [15].

The collected dataset were then trained and classified by using Bayesian Network (BN) before the results were verified by a set of questionnaire which were discussed comprehensively in [13].

In the system under study, there are two fundamental emotions and one blended emotion considered. The two fundamental emotions are happy (joy) and sad, while the blended emotion is nervous. On the other hand, natural or calm emotion is considered as a default emotion for the hybrid automata system. 


\subsection{Hybrid Automata and Robotic Platform}

The set of discrete input for the hybrid automata model is the ten EM signal values measured from the 10 body points that are eventually classified into a particular emotion. The output which is continuous in nature is the desired speed of the rehabilitation platform representing different rehabilitation tasks. The basic structure of the hybrid automata model of the system is shown in Figure 2 and can be can be described in 6-tuple as in Equation (1);

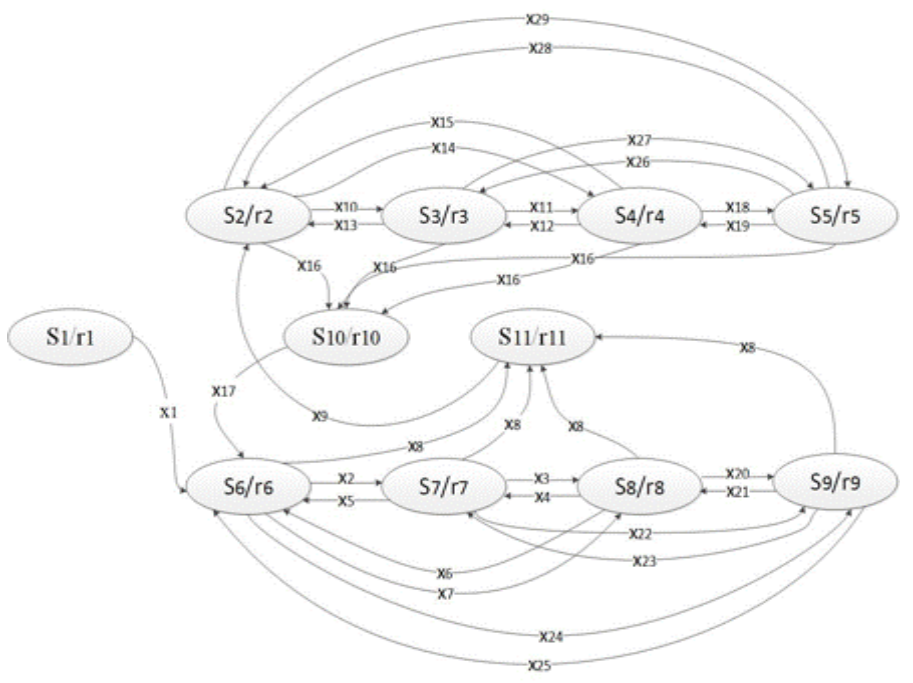

Figure 2: Hybrid Automata Model

Hybrid automata, $\mathrm{HA}=\left[\mathrm{X}, \Sigma, \mathrm{r}, f, \mathrm{X}_{\mathrm{o}}, \mathrm{X}_{\mathrm{m}}\right]$

where,

i. $\mathrm{X}=$ Control states $=\left\{S_{1}, S_{2}, S_{3}, S_{4}, S_{5}, S_{6}, S_{7}, S_{8}, S_{9}, S_{10}, S_{11}\right\}$

ii. $\Sigma=$ Plant symbol $=\left\{x_{1}, x_{2}, x_{3}, x_{4}, x_{5}, x_{6}, x_{7}, x_{8}, x_{9}, x_{10}, x_{11}, x_{12}, x_{13}, x_{14}, x_{15}, x_{16}, x_{17}, x_{18}, x_{19}, x_{20}, x_{21}, x_{22}, x_{23}, x_{24}, x_{25}\right.$, $\left.x_{26,}, x_{27}, x_{28}, x_{29}\right\}$

iii. $\mathrm{r}=$ Control symbol $=\left\{r_{1}, r_{2}, r_{3}, r_{4}, r_{5}, r_{6}, r_{7}, r_{8}, r_{9}, r_{10}, r_{11}\right\}$

iv. $f=$ Transition function $=\left\{f\left(S_{1}, x_{1}\right)=S_{6}, f\left(S_{6}, x_{2}\right)=S_{7}, f\left(S_{7}, x_{3}\right)=S_{8}, f\left(S_{8}, x_{4}\right)=S_{7}, f\left(S_{7}, x_{5}\right)=S_{6}, f\left(S_{8}, x_{6}\right)=S_{6}\right.$, $f\left(S_{6}, x_{7}\right)=S_{8}, f\left(S_{6}, x_{8}\right)=S_{11}, f\left(S_{7}, x_{8}\right)=S_{11}, f\left(S_{8}, x_{8}\right)=S_{11}, f\left(S_{9,} x_{8}\right)=S_{11}, f\left(S_{11}, x_{9}\right)=S_{2}, \quad f\left(S_{2}, x_{10}\right)=S_{3}, f\left(S_{3}, x_{11}\right)=S_{4}$, $f\left(S_{4}, x_{12}\right)=S_{3}, f\left(S_{3}, x_{13}\right)=S_{2}, f\left(S_{2}, x_{14}\right)=S_{4}, f\left(S_{4}, x_{15}\right)=S_{2}, f\left(S_{2}, x_{16}\right)=S_{10}, f\left(S_{3}, x_{16}\right)=S_{10}, f\left(S_{4}, x_{16}\right)=S_{10}, f\left(S_{5}, x_{16}\right)=S_{10}$ $f\left(S_{10}, x_{17}\right)=S_{6,} f\left(S 4_{3}, x_{18}\right)=S_{5}, f\left(S_{5}, x_{19}\right)=S_{4}, f\left(S_{8}, x_{20}\right)=S_{9}, f\left(S_{9}, x_{21}\right)=S_{8}, f\left(S_{7}, x_{22}\right)=S_{9}, f\left(S_{9}, x_{23}\right)=S_{7}, f\left(S_{6}, x_{24}\right)=S_{9}$, $\left.f\left(S_{9,}, x_{25}\right)=S_{6}, f\left(S_{5}, x_{26}\right)=S_{3}, f\left(S_{3}, x_{27}\right)=S_{5}, f\left(S_{5}, x_{28}\right)=S_{2}, f\left(S_{2}, x_{29}\right)=S_{5}\right\}$

v. $\quad \mathrm{X}_{0}=$ Initial state $=\left\{S_{1}\right\}$ and

vi. $\mathrm{X}_{\mathrm{m}}=$ Final state $=\left\{S_{11}\right\}$ 
Control state $\mathrm{X}$, is a finite set of control modes including the notable initial modes, $\mathrm{X}_{0}$ which is in this case labeled as $S_{1}$. Each of the modes represents the dynamic evolution of the system (i.e. speed, and direction of the platform and subject emotion) during the rehabilitation session. Plant symbol $\Sigma$, or the finite set of action in hybrid automata is used as the guard condition in the transition of the system from one mode to another mode. $r$ is control symbol represents the subset domains of the plant symbol consisting of flow and/or invariant conditions of each modes. These symbols are employed to represent the task assigned to the rehabilitation platform in changing the speed and direction of the gripper on the system based on the emotion of the subject. Transition function $f$, defines the transition from source mode $S$, to target mode $S$, dictated by the dynamic of state $x$. The final mode in the hybrid automata system stops the system operation and labeled as final state $\left(\mathrm{X}_{\mathrm{m}}\right)$ [16].

The controller modality for the developed automata is called Discrete Event System (DES) as shown in Figure 3. The DES behavior is governed by the occurrence of events, and the time evolves between each event occurs has no effect to the system. DES consists of three parts namely the high level controller (HLC), low level controller (LLC), and interface. The LLC part which usually represents the "Plant" is composed of set of low level controllers, the platform under control which also include human subject operating the platform. Meanwhile the HLC part is the brain of the system in-charge of discrete decision process programmed using hybrid automata framework [17]. The interface part acts as signal converter between continuous and discrete signals.

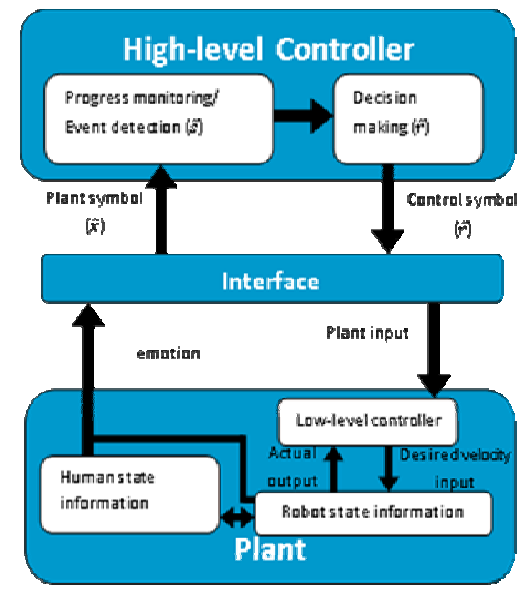

Figure 3: DES of Rehabilitation Platform with Emotion Embodiment System

The system under control (Figure 4) is a dedicated rehabilitation platform that is used to rehabilitate the affected upper limb. It consists of four parts which are linear guide actuator (part 1), gripper (part 2), locking mechanism (part 3), and rotational axis actuator (part 4). The linear actuator rehabilitates the affected arm by assisting the movement of the arm placed on the gripper back and forth repetitively while the unaffected arm is held constraint.

The controller of the platform is adaptive in deploying various rehabilitation tasks. Speed and direction of movement here are the variables to be controlled upon detection of specific discrete emotion. 


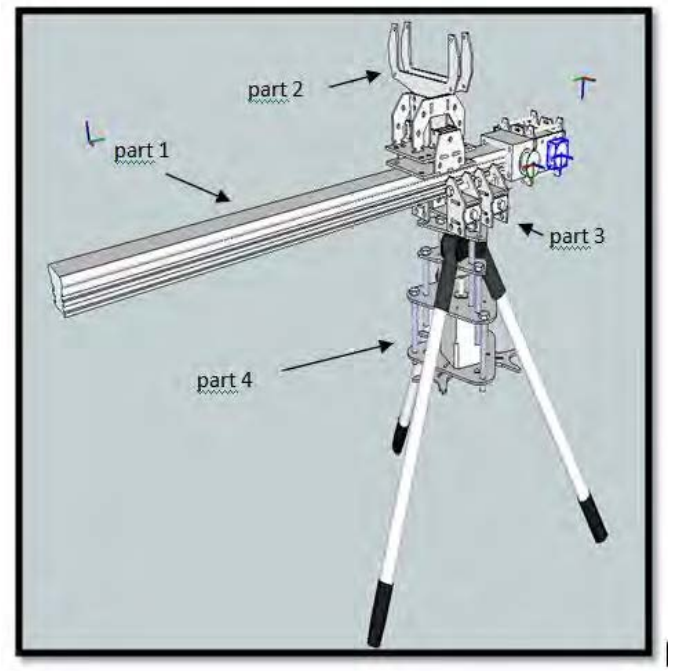

Figure 4: Model of Robot Assisted Rehabilitation Platform

\section{Hybrid Automata with Emotion Embodiment}

The scope of this paper is to present a hybrid automata model to control the operation of a robot assisted rehabilitation platform. The continuous set of platform dynamics is governed by the discrete emotions. The intention here is to mimic the human therapist in adapting the type of rehabilitation tasks based on the emotional state of the subject or patient. This enables the system to work more naturally in autonomous form when the input from the muscle health measurement is also considered. However the issue of muscle health is beyond the scope of this paper.

The input to the system is the subject's emotion, $E$. The position of the gripper, $X(\mathrm{~cm})$ is obtained from potentiometer reading on the platform, and from that the speed, $v$ of the gripper $(\mathrm{cm} / \mathrm{s})$, and the direction, dir the gripper moves are derived. The desired speed that is set for the gripper to move depends on the automaton that is triggered by the respective emotion [18]. The operation of the system is defined as different tasks of linear actuator or gripper to move at different speeds and directions when the affected arm is rehabilitated. Table 1 and Table 2 show the list of control states and control symbols for robot assisted rehabilitation platform respectively. Control states represent different dynamics of the platform modes labeled by Intro, Medium and Advanced in order to represent different speeds and directions of the movement. Meanwhile control symbol specifies the desired speed in which the platform is moving the arm.

Table 1: List of control states and platform modes

\begin{tabular}{|c|c|}
\hline Control State & Platform Modes \\
\hline State $1\left(S_{1}\right)$ & Initial \\
\hline State $2\left(S_{2}\right)$ & Intro Mode Back \\
\hline State $3\left(S_{3}\right)$ & Medium Mode Back \\
\hline State $4\left(S_{4}\right)$ & Advance Mode Back \\
\hline State $5\left(S_{5}\right)$ & Rilex Mode Back \\
\hline State $6\left(S_{6}\right)$ & Intro Mode Forth \\
\hline State $7\left(S_{7}\right)$ & Medium Mode Forth \\
\hline State $8\left(S_{8}\right)$ & Advance Mode Forth \\
\hline
\end{tabular}




\begin{tabular}{|c|c|}
\hline State $9\left(S_{9}\right)$ & Rilex Mode Forth \\
\hline State $10\left(S_{10}\right)$ & End Forth \\
\hline State $11\left(S_{11}\right)$ & End Back \\
\hline
\end{tabular}

Table 2: List of control symbol and task assigned

\begin{tabular}{|c|c|}
\hline Control Symbol & Task Assigned \\
\hline$r_{1}$ & The gripper moves backward to home position $(X=20)$ with maximum speed of $1 \mathrm{~cm} / \mathrm{s}$ \\
\hline$r_{2}$ & Subject is sad $(v=0.5 \mathrm{~cm} / \mathrm{s}$ and $\operatorname{dir}=$ backward $)$ \\
\hline$r_{3}$ & Subject is nervous $(v=0.667 \mathrm{~cm} / \mathrm{s}$ and $d i r=$ backward $)$ \\
\hline$r_{4}$ & Subject is happy $(v=1.0 \mathrm{~cm} / \mathrm{s}$ and $d i r=$ backward $)$ \\
\hline$r_{5}$ & Subject is calm $(v=0.833 \mathrm{~cm} / \mathrm{s}$ and dir $=$ backward $)$ \\
\hline$r_{6}$ & Subject is sad $(v=0.5 \mathrm{~cm} / \mathrm{s}$ and $\operatorname{dir}=$ forward $)$ \\
\hline$r_{7}$ & Subject is nervous $(v=0.667 \mathrm{~cm} / \mathrm{s}$ and $\mathrm{dir}=$ forward $)$ \\
\hline$r_{8}$ & Subject is happy $(v=1.0 \mathrm{~cm} / \mathrm{s}$ and $\operatorname{dir}=$ forward $)$ \\
\hline$r_{9}$ & Subject is calm $(v=0.833 \mathrm{~cm} / \mathrm{s}$ and $\operatorname{dir}=$ forward $)$ \\
\hline$r_{10}$ & $\begin{array}{l}\text { Negate dir value (change direction from forward to backward with minimum } \\
\qquad v=0.333 \mathrm{~cm} / \mathrm{s} \text { ) }\end{array}$ \\
\hline$r_{11}$ & $\begin{array}{l}\text { Negate } \operatorname{dir} \text { value (change direction from backward to forward with minimum } \\
\qquad v=0.333 \mathrm{~cm} / \mathrm{s} \text { ) }\end{array}$ \\
\hline
\end{tabular}

The plant symbols listed in Table 3 are generated based on the information from position sensor of the robotic platform and the emotion of the subject. It represents the possible evolution of system dynamics in terms of speed, direction and the subject emotions. As these dynamics changes, new plant symbol is generated when the requirement of the guard condition of the hybrid automata is fulfilled.

Table 3: List of plant symbol and definition

\begin{tabular}{|c|c|}
\hline Plant Symbol & Type of Emotion and Gripper position ( $X$ in $\mathrm{cm})$ \\
\hline$x_{1}$ & $X=20$ \\
\hline$x_{2}$ & Subject emotion $E$, changes from sad to nervous AND $(X=5)$ \\
\hline$x_{3}$ & Subject emotion $E$, changes from nervous to happy AND $(X=5)$ \\
\hline$x_{4}$ & Subject emotion $E$, changes from happy to nervous AND $(X=5)$ \\
\hline$x_{5}$ & Subject emotion $E$, changes from nervous to sad AND $(X=5)$ \\
\hline$x_{6}$ & Subject emotion $E$, changes from happy to sad AND $(\mathrm{X}>=5)$ \\
\hline$x_{7}$ & Subject emotion $E$, changes from sad to happy AND (X>=5) \\
\hline$x_{8}$ & Gripper at goal position $(X=5)$ \\
\hline$x_{9}$ & Gripper direction dir, changes from forward to backward $(5<X<6)$ \\
\hline$x_{10}$ & Subject emotion $E$, changes from sad to nervous AND $(X<=20)$ \\
\hline$x_{11}$ & Subject emotion $E$, changes from nervous to happy AND $(X<=20)$ \\
\hline$x_{12}$ & Subject emotion $E$, changes from happy to nervous AND $(X<=20)$ \\
\hline$x_{13}$ & Subject emotion $E$, changes from nervous to sad AND $(X<=20)$ \\
\hline$x_{14}$ & Subject emotion $E$, changes from sad to happy AND $(X<=20)$ \\
\hline$x_{15}$ & Subject emotion $E$, changes from happy to sad AND $(X<=20)$ \\
\hline$x_{16}$ & Gripper at home position $(X=20)$ \\
\hline$x_{17}$ & Gripper direction dir, changes from backward to forward $(19<X<20)$ \\
\hline$x_{18}$ & Subject emotion $E$, changes from happy to calm AND $(X<=20)$ \\
\hline$x_{19}$ & Subject emotion $E$, changes from calm to happy AND $(X<=20)$ \\
\hline$x_{20}$ & Subject emotion $E$, changes from happy to calm AND $(X=5)$ \\
\hline$x_{21}$ & Subject emotion $E$, changes from calm to happy AND $(X=5)$ \\
\hline$x_{22}$ & Subject emotion $E$, changes from nervous to calm AND $(X=5)$ \\
\hline$x_{23}$ & Subject emotion $E$, changes from calm to nervous AND $(X=5)$ \\
\hline$x_{24}$ & Subject emotion $E$, changes from sad to calm AND $(X=5)$ \\
\hline
\end{tabular}




\begin{tabular}{|c|c|}
\hline$x_{25}$ & Subject emotion $E$, changes from calm to sad AND $(X=5)$ \\
\hline$x_{26}$ & Subject emotion $E$, changes from calm to nervous AND $(X<=20)$ \\
\hline$x_{27}$ & Subject emotion $E$, changes from nervous to calm AND $(X<=20)$ \\
\hline$x_{28}$ & Subject emotion $E$, changes from calm to sad AND $(X<=20)$ \\
\hline$x_{29}$ & Subject emotion $E$, changes from sad to calm AND $(X<=20)$ \\
\hline
\end{tabular}

\section{Results and Discussion}

The system employs Arduino microcontroller as data acquisition system (DAQ) between the position sensor and actuators of the robotic platform. A computer equipped with Matlab/Simulink software is used to model the hybrid automata system. The system is developed using Stateflow tool in Simulink and the data/control interaction is done through Arduino support package, Arduino IO [19]. The 'Arduino Analog Read' block is selected to record the value of the potentiometer reading, $X$ in order to get the exact position of the gripper when it moves. The 'Arduino Digital Write' block functions as the main switch for the hybrid automata in changing states while 'Arduino Analog Write' is used to command the DC motor to actuate according to the preset desired speed $v$ in the respected automaton, and finally, the 'Flag' block is used to control the direction dir of the gripper.

To study the efficacy and the performance of the hybrid automata system, a case study is designed to reflect the possible events that might occur in the real setup. The emotion recognition system takes the subject emotion, $E$ in real time. A constant block is utilized as in integrating the Simulink-Stateflow with rehabilitation platform and the emotion of the subject [20]. As a result, the ordered sequence of subject emotions induced is given as a shown in Equation (2);

$E=\left\{\begin{array}{lllll}1 & 2 & 0 & 2 & 3\end{array}\right\}$

where $E=0$ (calm), $E=1$ (sad), $E=2$ (nervous), and $E=3$ (happy).

In the case study, the initial emotion of the subject is $\operatorname{sad}(E=1)$ at the beginning of the rehabilitation session. When the task execution starts, state $S_{l}$ is triggered since the Stateflow is in initialization mode. The gripper is moving backward to the home position as part of system initialization with the desired speed of $1 \mathrm{~cm} / \mathrm{s}$ (point A in Figure 8). When the subject's arm has reached the home position $(X=20 \mathrm{~cm})$ at point B, state $S_{6}$ is activated to guide the patient arm's movement in forward motion with the desired speed of $-0.5 \mathrm{~cm} / \mathrm{s}$.

At point $\mathrm{C}$, the emotion changes to nervous $(E=2)$, causing the gripper desired speed changes to $-0.667 \mathrm{~cm} / \mathrm{s}$ and the active state is switches to state $S_{7}$. As the gripper reaches the goal position $(X=5 \mathrm{~cm})$ at point $\mathrm{D}$ for the first time, the emotion detected is still nervous $(E=2)$, but the state is now switched to $S_{11}$ to prepare the gripper to change direction at speed of $0.333 \mathrm{~cm} / \mathrm{s}$. At point $\mathrm{E}$, the emotion remain nervous $(E=2)$, prompting the state to switch to state $S_{3}$ and the desired speed is set to to $0.667 \mathrm{~cm} / \mathrm{s}$. On moving the gripper backward, the subject emotion is induced to calm $(E=0)$ as shown at point $\mathrm{F}$, thus the desired speed adapts to $0.833 \mathrm{~cm} / \mathrm{s}$ and state $S_{5}$ becomes is activated.

At point $\mathrm{G}$, the state switches to state $S_{3}$ due to the change in the emotion again to nervous $(E=2)$ and the desired speed of the gripper is changing to $0.667 \mathrm{~cm} / \mathrm{s}$. At point $\mathrm{H}$, when the home position is reached $(X=20 \mathrm{~cm})$, the speed negates to $-0.333 \mathrm{~cm} / \mathrm{s}$ when state $S_{10}$ is activated. It symbolizes that the subject's arm has reached the home position and the gripper direction is changed to forward motion again.

At point I, the state switches to state $S_{7}$ denoting the emotion detected is still nervous ( $\left.E=2\right)$, thus the desired speed is set to $-0.667 \mathrm{~cm} / \mathrm{s}$. At the final stage the subject is detected to be in the happy emotion $(E=3)$ as can be seen at point J. It activates $S_{8}$ with the speed of $-1.0 \mathrm{~cm} / \mathrm{s}$. 

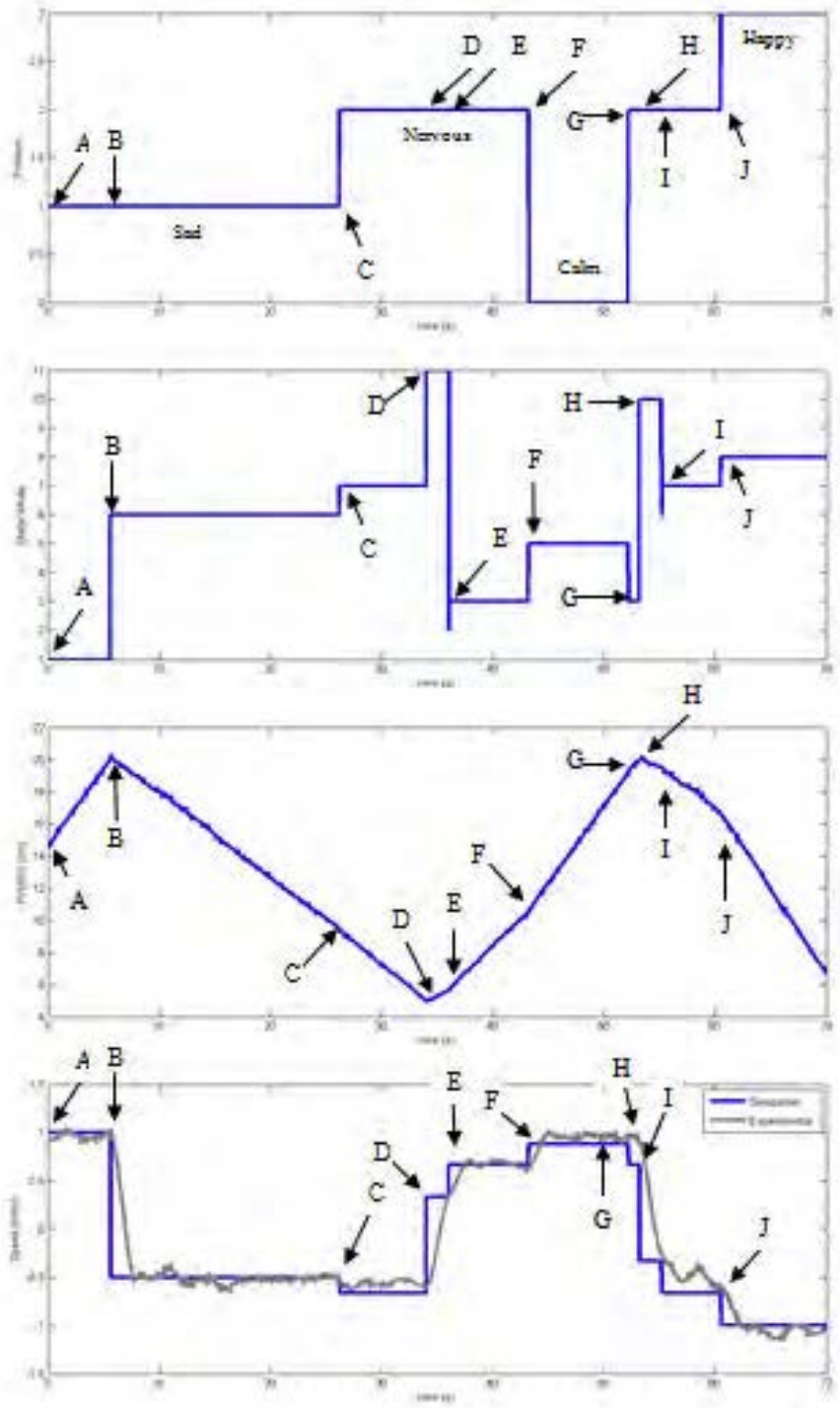

Figure 8: Emotion, State, Position, and Speed Profiles of Rehabilitation Platform

\section{Conclusion}

In this paper, a hybrid automata model is developed and tested on robot-assisted rehabilitation platform for upper-extremity. The proposed hybrid automata model embodies the emotion of the subject which results in deployment of different rehabilitation tasks. The experimental results show the efficacy of hybrid automata model to be applied on a rehabilitation platform with different type of emotions as input to the system. For the future work, the model of hybrid automata can be improved to include the degree of probability and time of the emotions as well as the recovery level of the affected muscle. The stability of the controller can also be investigated to ensure the system is stable throughout the switching between modes in the hybrid automata model. 


\section{Acknowledgement}

The work presented was carried out in the Biomechatronics Research Laboratory of International Islamic University Malaysia. The authors wish to acknowledge the subjects who took part in this project and grant funding from the Ministry of Higher Education Malaysia (RAGS 12-002-0002).

\section{References}

[1] T. A. Henzinger, The theory of hybrid automata: Springer, 2000.

[2] J.-F. Raskin, "An introduction to hybrid automata," in Handbook of networked and embedded control systems, ed: Springer, 2005, pp. 491-517.

[3] K. Kumar and M. V. Vaidyan, "Modeling of Non-convex Obstacle Detection and Avoidance Mobile Robot by Hybrid Cellular Automata," in Advances in Computing and Communications (ICACC), 2013 Third International Conference on, 2013, pp. 9-12.

[4]H. Fu, G. Tian, Q. Chen, and Y. Jin, "Hybrid automata of an integrated motor-transmission powertrain for automatic gear shift," in American Control Conference (ACC), 2011, 2011, pp. 4604-4609.

[5] A. Schwarze, M. Buntins, J. Schicke-Uffmann, U. Goltz, and F. Eggert, "Modelling driving behaviour using hybrid automata," IET Intelligent Transport Systems, vol. 7, pp. 251-256, 2013.

[6] Y. Ge, Y. Chen, and G. Zhang, "Longitudinal control of intelligent vehicle based on hybrid automata model," in Intelligent Control and Automation (WCICA), 2012 10th World Congress on, 2012, pp. 1848-1853.

[7] Y. Yalei and Z. Xingshe, "Cyber-Physical Systems Modeling Based on Extended Hybrid Automata," in Computational and Information Sciences (ICCIS), 2013 Fifth International Conference on, 2013, pp. 1871-1874.

[8] A. He and J. Wang, "A formal analysis of stage stunt system with hybrid automata," in Computing and Convergence Technology (ICCCT), 2012 7th International Conference on, 2012, pp. 741-744.

[9] G. Caravagna, A. d'Onofrio, M. Antoniotti, and G. Mauri, "Stochastic Hybrid Automata with delayed transitions to model biochemical systems with delays," Information and Computation, vol. 236, pp. 19-34, 2014.

[10] S. N. Sidek, A. U. Shamsudin, and E. Ismail, "A Hybrid Controller with Chedoke-McMaster Stroke Assessment for Robot-Assisted Rehabilitation," Procedia Engineering, vol. 41, pp. 629-635, 2012.

[11] Y.-M. Kim and D.-S. Kwon, "A fuzzy intimacy space model to develop human-robot affective relationship," in World Automation Congress (WAC), 2010, 2010, pp. 1-6.

[12] J. Ma, Y. Zhang, Y. Nam, A. Cichocki, and F. Matsuno, "EOG/ERP hybrid 
human-machine interface for robot control," in Intelligent Robots and Systems (IROS), 2013 IEEE/RSJ International Conference on, 2013, pp. 859-864.

[13] C. R. Guerrero, J. F. Marinero, J. P. Turiel, and P. R. Farina, "Using psychophysiological feedback to enhance physical human robot interaction in a cooperative scenario," in Biomedical Robotics and Biomechatronics (BioRob), 2012 4th IEEE RAS \& EMBS International Conference on, 2012, pp. 1176-1181.

[14] A. S. Ghazali, S. Sidek, and S. Wok, "Affective State Classification using Bayesian Classifier," in IEEE Fifth International Conference on Intelligent System, Modelling and Simulation (ISMS2014) Langkawi, Malaysia, 2014.

[15] A. S. Ghazali and S. N. i. Sidek, "Non-invasive non-contact based affective state identification," in Computer Applications and Industrial Electronics (ISCAIE), 2014 IEEE Symposium on, 2014, pp. 1-5.

[16] R. Alur, "Formal verification of hybrid systems," in Embedded Software (EMSOFT), 2011 Proceedings of the International Conference on, 2011, pp. 273-278.

[17] E. Dugyun and N. Sarkar, "A High-Level Controller For Robot-Assisted Rehabilitation," in Procedding the IEEE 28th EMBS Annual International Conference, 2006.

[18] S. N. Sidek, A. S. Ghazali, and S. Wok, "Emotion Embodiment in Robot-Assisted Rehabilitation System Using Hybrid Automata," in Advanced Applied Informatics (IIAIAAI), 2014 IIAI 3rd International Conference on, 2014, pp. 505-509.

[19] M. Central. (2013, February, 27). MATLAB Support Package for Arduino (aka ArduinoIO Package). Available: http://www.mathworks.com/matlabcentral/fileexchange/32374-matlab-support-packagefor-arduino-aka-arduinoio-package

[20] A. S. Ghazali and S. N. Sidek, "Electromagnetic Based Emotion Recognition Using ANOVA Feature Selection and Bayes Network," in 2014 IEEE Conferece on Biomedical Engineering and Sciences, 2014. 